\title{
Formação do rio transcontinental do Amazonas guiada por processos tectônicos e processos superficias de erosão e sedimentação.
}

Tacio Cordeiro Bicudo \& Victor Sacek, Instituto de Astronomia, Geofísica e Ciências Atmosféricas, USP, São Paulo, Brasil.

Copyright 2016, SBGf - Sociedade Brasileira de Geofísica

Este texto foi preparado para a apresentação no VII Simpósio Brasileiro de Geofísica, Ouro Preto, 25 a 27 de outubro de 2016. Seu conteúdo foi revisado pelo Comitê Técnico do VII SimBGf, mas não necessariamente representa a opinião da SBGf ou de seus associados. É proibida a reprodução total ou parcial deste material para propósitos comerciais sem prévia autorização da SBGf.

\section{Resumo}

During the Cenozoic, the Andean orogeny induced a great change in drainage pattern in northern South America, culminating in the development of the Amazon River as a transcontinental river. Through simulations using a numerical model, we studied the evolution of the landscape focusing on the onset of the Amazon River. The numerical model accounts for the interaction of orogeny, flexure and isostasy of the lithosphere and surface processes of erosion and sedimentation. In all the different numerical scenarios simulated, it was possible to reproduce the change in the drainage pattern culminating in the formation of the Amazon River followed by the increase in the sedimentation rate in the Amazon Fan. We also analyzed the influence of the effective elastic thickness of the lithosphere $T_{e}$ and the crustal thickening rate of the andean region in the evolution of the landscape. We concluded that large values of $T_{e}$ induces a delay in the reversal of the drainage pattern. However, fast uplift rates of the Andes result in a fast reorganization of the drainage in the northern South America, anticipating the formation of the transcontinental Amazon River.

\section{Introdução}

A bacia hidrográfica do Rio Amazonas abrange uma área aproximada de $7 \times 10^{6} \mathrm{~km}^{2}$ do norte da América do Sul. $\mathrm{O}$ seu canal mais longo, com quase $7.000 \mathrm{~km}$, tem origem nos Andes peruanos e cruza todo o continente até desaguar na Foz do Amazonas, que fica no Atlântico, na costa equatorial brasileira.

Porém, a data de formação dessa grande bacia hidrográfica e como se deu a evolução da paisagem na Amazônia ainda são debatidos (Hoorn et al., 2010; Latrubesse et al., 2010), assim como os processos que guiaram essa evolução (Shephard et al., 2010; Sacek, 2014).

A tectônica andina teve protagonismo na evolução da paisagem na Amazônia, que resultou em uma mudança no padrão de drenagem e na formação da bacia hidrográfica do Rio Amazonas como conhecemmos hoje (Hoorn et al.,1995; Hoorn et al., 2010).

Antes do início do soerguimento dos Andes, o Cráton Amazônico era a principal fonte de sedimentos para as bacias sedimentares no norte da América do Sul. Os altos estruturais no embasamento separavam as bacias sedimentares e as bacias de drenagem. Um importante alto estrutural que separa a Bacia do solimões da Bacia do Amazonas é o Alto de Purus (Wanderley-Filho et al., 2010). A leste do alto estrutural existia um pequeno sistema de rios com origem no Cráton que desaguava bem próxima da foz do Rio Amazonas de hoje (Hoor et al., 2010; Figueiredo et al., 2010), a oeste os rios também tinham origem no Cráton mas eram drenados para o Caribe ou até para o Oceano Pacífico (Hoorn et al., 2010)

Hoorn et al., (2010) propuseram que a bacia hidrográfica do Amazonas tomou essas proporções apenas no final do Mioceno, e que até então no oeste amazônico existia uma grande zona alagada, denominada por esses autores como Sistema Pebas. Essa data foi proposta por Figueiredo et al. (2009) que estudaram dois poços situados no leque do Amazonas, sugerindo 0 estabelecimento do rio transcontinental no final do Mioceno, entre 11,8 e 11,3 milhões de anos atrás, quando é observado na coluna estratigráfica um aumento significativo na taxa de deposição sedimentar.

Por outro lado, Latrubesse et al., (2010) propõe que até o inicio do Plioceno ( 5,3 Ma) um sistema de mega-leque existia no oeste amazônico e era limitado a leste pelo Alto de Purus. Ele explica que o aumento na taxa de sedimentação observado por Figueiredo et al., (2009) no final do Mioceno deve-se ao aumento do aporte de sedimentos provenientes do Cráton e não dos Andes.

Nesse trabalho usaremos a modelagem numérica conjunta de diferentes processos para dar vínculos quantitaitvos aos diferentes cenários propostos para a formação do Rio Amazonas.

\section{Metodologia}

O modelo numérico usado nesse trabalho foi desenvolvido por Sacek (2014) e inclui os seguintes processos: 1) orogenia; 2) flexura e isostasia da litosfera; 3) clima (precipitação); 4) processos superficiais (erosão e sedimentação).

A orogenia é simulada através de um espessamento crustal. Esse é um processo que não é afetado pelos outros, ou seja, a taxa de espessamento crustal $\dot{h}_{o r}(x, y, t)=\frac{\partial h}{\partial t}$ não é modificada por nenhum outro processo, é imposta no início da simulação. Em certo intervalo de tempo $\Delta t$, o espessamento crustal eleva a topografia $h \leftarrow h+\Delta t \dot{h}_{o r}$ e induz uma carga vertical na litosfera $F_{o r}=-\rho_{c} g \Delta t \dot{h}_{o r}$, onde $g$ é a aceleração da gravidade.

As cargas na litosfera $F=F_{s p}+F_{o r}$ podem ser orogênicas $F_{o r}$ ou sedimentares $F_{s p}$ e são compensadas tanto pela rigidez da litosfera quanto pelo empuxo da astenosfera. A equação 1 descreve o comportamento de uma placa elástica fina flutuando em um líquido invíscido 
sobre a ação de uma força vertical e foi solucionada numericamente por Sacek e Ussami (2009) através do método do elemento finito.

$$
(\boldsymbol{L} \boldsymbol{\nabla}) \boldsymbol{D} \boldsymbol{L} \boldsymbol{\nabla} w+\left(\rho_{m}-\rho_{\text {in }}\right) g w=F,
$$

onde $w$ é o deslocamento vertical, $\rho_{m}$ é a densidade do manto, $\rho_{\text {in }}$ a densidade do sedimento, o operador $(\boldsymbol{L} \boldsymbol{V})$ é descito na equação 2. E $\boldsymbol{D}$ é uma matriz $3 \times 3$ (equação 3 ), função da razão de Poisson $v$ e da rigidez $D$ (equação 4).

$$
\begin{gathered}
(\boldsymbol{L} \boldsymbol{\nabla})=\left[\frac{\partial^{2}}{\partial x^{2}}, \frac{\partial^{2}}{\partial y^{2}}, 2 \frac{\partial^{2}}{\partial x \partial y}\right]^{T} \\
\boldsymbol{D}=D\left[\begin{array}{ccc}
1 & v & 0 \\
v & 1 & 0 \\
0 & 0 & (1-v) / 2
\end{array}\right] \\
D=E\left(T_{e}\right)^{3} / 12\left(1-v^{2}\right),
\end{gathered}
$$

onde $T_{e}$ é a espessura elástica efetiva da litosfera e $E$ é o módulo de Young.

A precipitação sobre o modelo é constante $V_{R}=1 \mathrm{~m} /$ ano e alimenta os rios que por sua vez redistribuem as massas na superfície. A Figura 1 traz um fluxograma de como os processos interagem entre si.

O transporte fluvial acontece da região de maior topografia para a região adjacente de menor topografia e com maior módulo de gradiente topográfico $|\nabla h|$. A quantidade de sedimentos que uma região é capaz de transmitir é dada pela equação 5 :

$$
q_{f}^{e q b}=k_{f} q_{r}{ }^{m^{\prime}}|\nabla h|^{n^{\prime}}
$$

onde $k_{f}$ é o coeficiente de transporte fluvial e $q_{r}$ é a descarga d'água cumulativa proveniente das regiões de maior topografia mais a precipitação sobre a região. Os valores dos expoentes $m$ ' e $n$ ' adotados nesse trabalho são ambos iguais a 1, assim como em Sacek (2014).

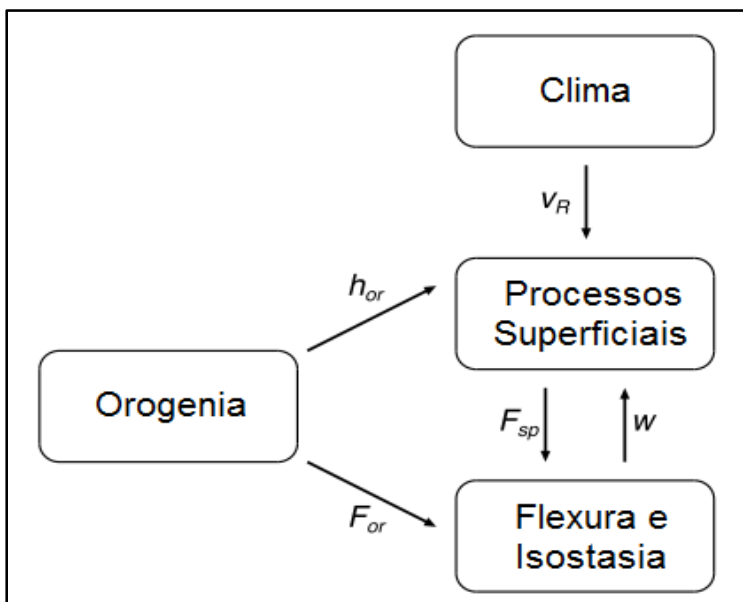

Figura 1:Fluxograma com o processos levados em conta no modelo e como eles interagem entre si.
O transporte fluvial causa erosão e deposição sedimentar, gerando uma variação na topografia:

$$
\frac{\partial h}{\partial t}=-\frac{q_{f}^{e q b}-q_{f}}{l_{f}},
$$

onde $q_{f}$ é o fluxo de sedimentos através da região e $l_{f}$ é a erodibilidade da rocha, quanto maior essa grandeza mais difícil a rocha é erodida.

O transporte de sedimentos offshore é simulado através de uma difusão não linear, onde o coeficiente de difusão $k_{m}=k_{m}^{*} \exp \left(\frac{h-h_{s . l .}}{h_{0}}\right)$ aumenta exponencialmente com a profundidade e é função de $k_{m}^{*}$, que é um coeficiente constante, $h_{\text {s.l. }}$ que é o nível do mar e é mantido constante e $h_{0}$ um fator de decaimento do transporte no mar. A variação na topografia devido ao transporte de sedimentos offshore

$\frac{\partial h}{\partial t}=\nabla \cdot\left(k_{m} \nabla h\right)$.

Para criar o cenário inicial para as simulações usamos como base a ilustração da Figura $1 \mathrm{~A}$ em Hoorn et al. (2010), que representa o norte da América do Sul antes do início do soerguimento dos Andes naquela região. Além disso foi usado um mapa de satélite da região. O cenário criado está ilustrado na Figura 2.

A taxa de espessamento crustal adotada nas simulações foram multiplicadas por um fator de escala $U$, assim como fez Sacek (2014) em seu trabalho.

Nos testes apresentados nesse resumo analisamos a sensibilidade do modelo à variação da taxa de espessamento crustal $\boldsymbol{U}$ e espessura elástica efetiva da litosfera $T_{e}$. Os valores de $T_{e}$ e $U$ que usamos para os teste estão na Tabela 1, juntamente com os valores de outros parâmetros do modelo. Testamos as 12 combinações possíveis de $T_{e}$ e $U$.

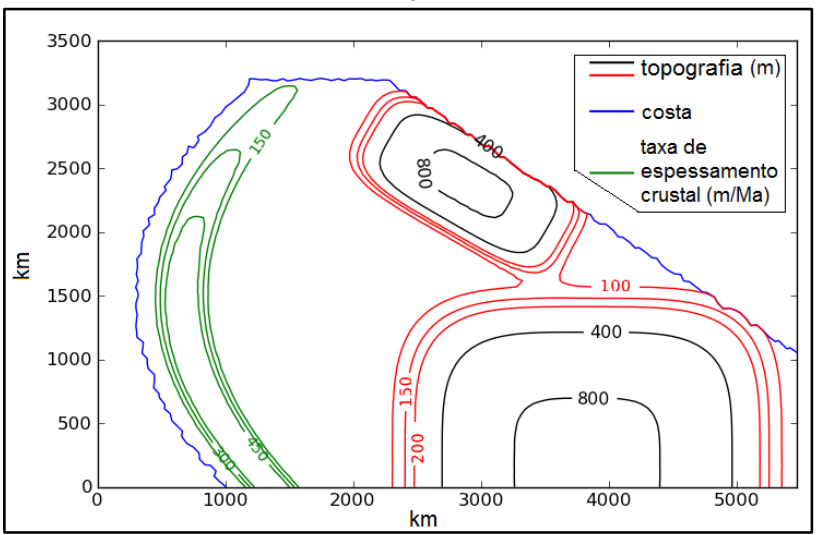

Figura 2:Cenário inicial testado nas simulações, a topografia nas planícies é $40 \mathrm{~m}$ e no mar $-4000 \mathrm{~m}$. As regiões de maior topografia no continente representam 0 Cráton Amazônico. 
Em seu trabalho, Sacek (2014), considera em seu modelo a precipitação orográfica, que leva em conta a topografia para fazer o cálculo do fluxo de água sobre o modelo. Nos testes apresentados aqui esse efeito não é levado em conta. Outra simplificação adotada foi quanto a espessura elástica efetiva da litosfera que também é constante em toda área do modelo.

O tempo máximo simulado nos cenários foi de 80 milhões de anos.

\section{Resultados e discussão}

Em todos os cenários testados foi possível observar a mudança no padrão de drenagem que corresponde formação do rio transcontinental Amazonas. Na Figura 3 é mostrada a evolução da topografia e das bacias de drenagem para um dos cenários testados.

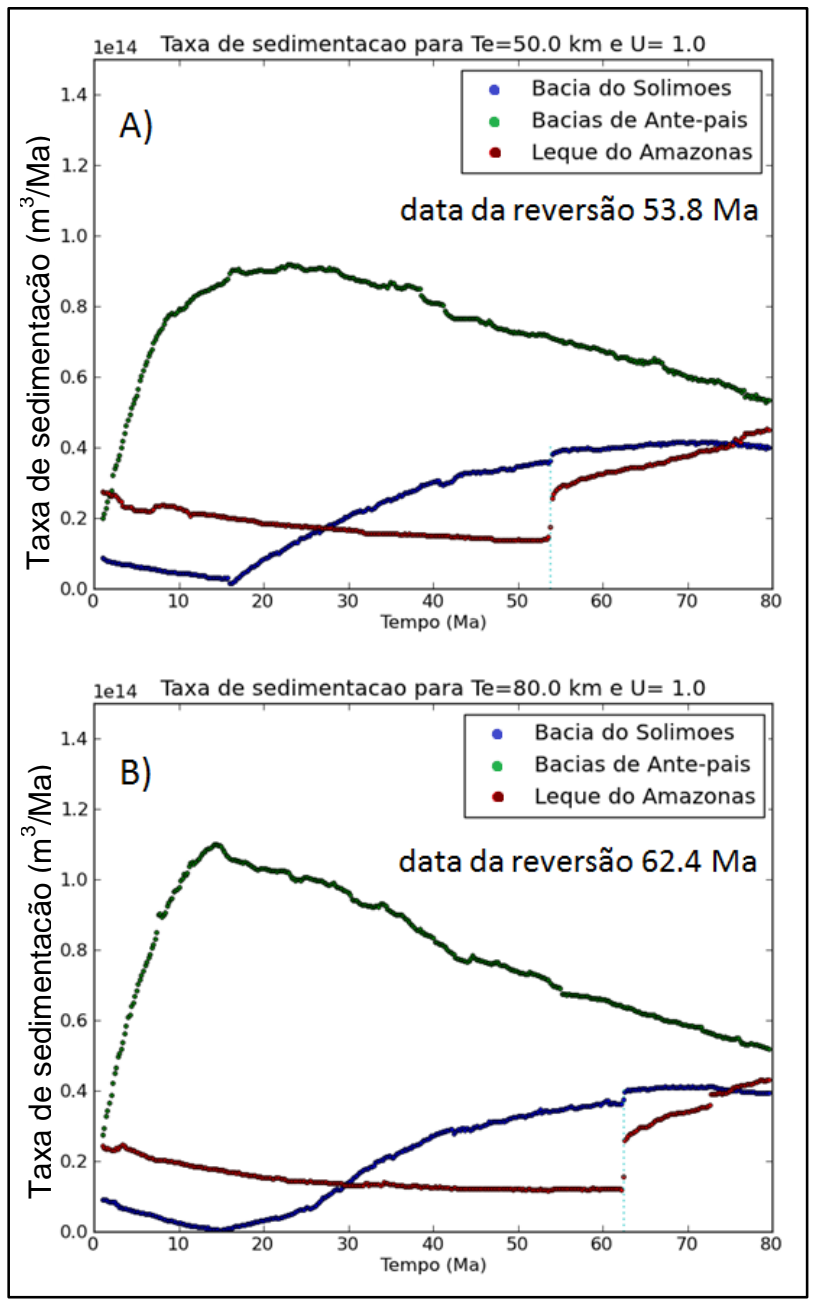

Figura 4:Taxa de sedimentação nas bacias sedimentares da região para dois cenários testados. A linha pontilhada azul marca a data em que ocorre maior variação na taxa de sedimentação na foz do Amazonas, essa data corresponde a data da mudança da drenagem nas simulações. O tempo mostrado é o tempo após o início da simulação.
Nos primeiros milhões de anos (Myr) após o início das simulações ocorre o deslocamento do divisor de drenagem para oeste. Isso ocorre devido a deposição de sedimentos do Cráton a oeste do divisor. Ao mesmo tempo, no oeste amazônico ocorre o desenvolvimento das bacias de ante-país, onde se forma um grande lago adjacente à cordilheira que é drenado para o caribe. As bacias de ante-país são rapidamente preenchidas com os sedimentos provenientes da cordilheira em desenvolvimento e esse lago é assoreado, formando um canal axial que continua sendo drenado para o caribe (veja Figura $3 \mathrm{~A}, \mathrm{~B}$ e C). Esse canal é progressivamente deslocado para leste e atinge a região equivalente à bacia do Solimões (veja Figura 3 E, F, G, H). Nesse tempo é observado um grande aumento da taxa de sedimentação na bacia do Solimões e uma diminuição na taxa nas bacias de ante-país (ver Figura 4).

Tabela 1:Valores dos parâmetros usados nos cenários testados juntamente com sua descrição.

\begin{tabular}{|c|c|c|c|}
\hline & âmetro & Descrição & valor \\
\hline \multirow{7}{*}{$\begin{array}{l}\text { Processos } \\
\text { Superficias }\end{array}$} & $k_{f}$ & $\begin{array}{l}\text { Coeficiente de transporte } \\
\text { fluvial }\end{array}$ & 0.08 \\
\hline & $l_{s}$ & $\begin{array}{l}\text { Erodibilidade das rochas } \\
\text { sedimentares }\end{array}$ & $1 \times 10^{5} \mathrm{~m}$ \\
\hline & $l_{b}$ & $\begin{array}{l}\text { Erodibilidade do } \\
\text { embasamento }\end{array}$ & $4 \times 10^{5} \mathrm{~m}$ \\
\hline & $l_{b 2}$ & $\begin{array}{c}\text { Erodibilidade do Cráton } \\
\text { Amazônico } \\
\end{array}$ & $4 \times 10^{6} \mathrm{~m}$ \\
\hline & $h_{s .}$ & Nível do mar & $0 \mathrm{~m}$ \\
\hline & $k_{m}^{*} \mathrm{C}$ & $\begin{array}{l}\text { oeficiente de transporte no } \\
\text { mar }\end{array}$ & $20 \mathrm{~m}^{2} / \mathrm{ano}$ \\
\hline & $h_{0}$ & $\begin{array}{l}\text { fator de decaimento do } \\
\text { transporte no mar }\end{array}$ & $3000 \mathrm{~m}$ \\
\hline \multirow{8}{*}{$\begin{array}{l}\text { Flexura e } \\
\text { isostasia }\end{array}$} & $T_{e}$ & $\begin{array}{c}\text { Espessura elástica efetiva } \\
\text { da litosfera (4 valores } \\
\text { testados) }\end{array}$ & $\begin{array}{l}50 \mathrm{~km} \\
60 \mathrm{~km} \\
70 \mathrm{~km} \\
80 \mathrm{~km}\end{array}$ \\
\hline & $E$ & Módulo de young & $10^{11} \mathrm{~N} / \mathrm{m}^{2}$ \\
\hline & $g$ & Aceleração da gravidade & $9,8 \mathrm{~m} / \mathrm{s}^{2}$ \\
\hline & $v$ & Razão de Poison & 0,25 \\
\hline & $\rho_{m}$ & Densidade do manto & $3300 \mathrm{~kg} / \mathrm{m}^{3}$ \\
\hline & $\rho_{c}$ & Densidade da crosta & $2700 \mathrm{~kg} / \mathrm{m}^{3}$ \\
\hline & $\rho_{s}$ & Densidade do sedimento & $2700 \mathrm{~kg} / \mathrm{m}^{3}$ \\
\hline & $\rho_{w}$ & Densidade da água & $1030 \mathrm{~kg} / \mathrm{m}^{3}$ \\
\hline Orogenia & $U$ & $\begin{array}{l}\text { Fator de escala da taxa de } \\
\text { espessamento crustal } \\
\text { (3 valores testados) }\end{array}$ & $\begin{array}{l}100 \% \\
200 \% \\
300 \%\end{array}$ \\
\hline
\end{tabular}




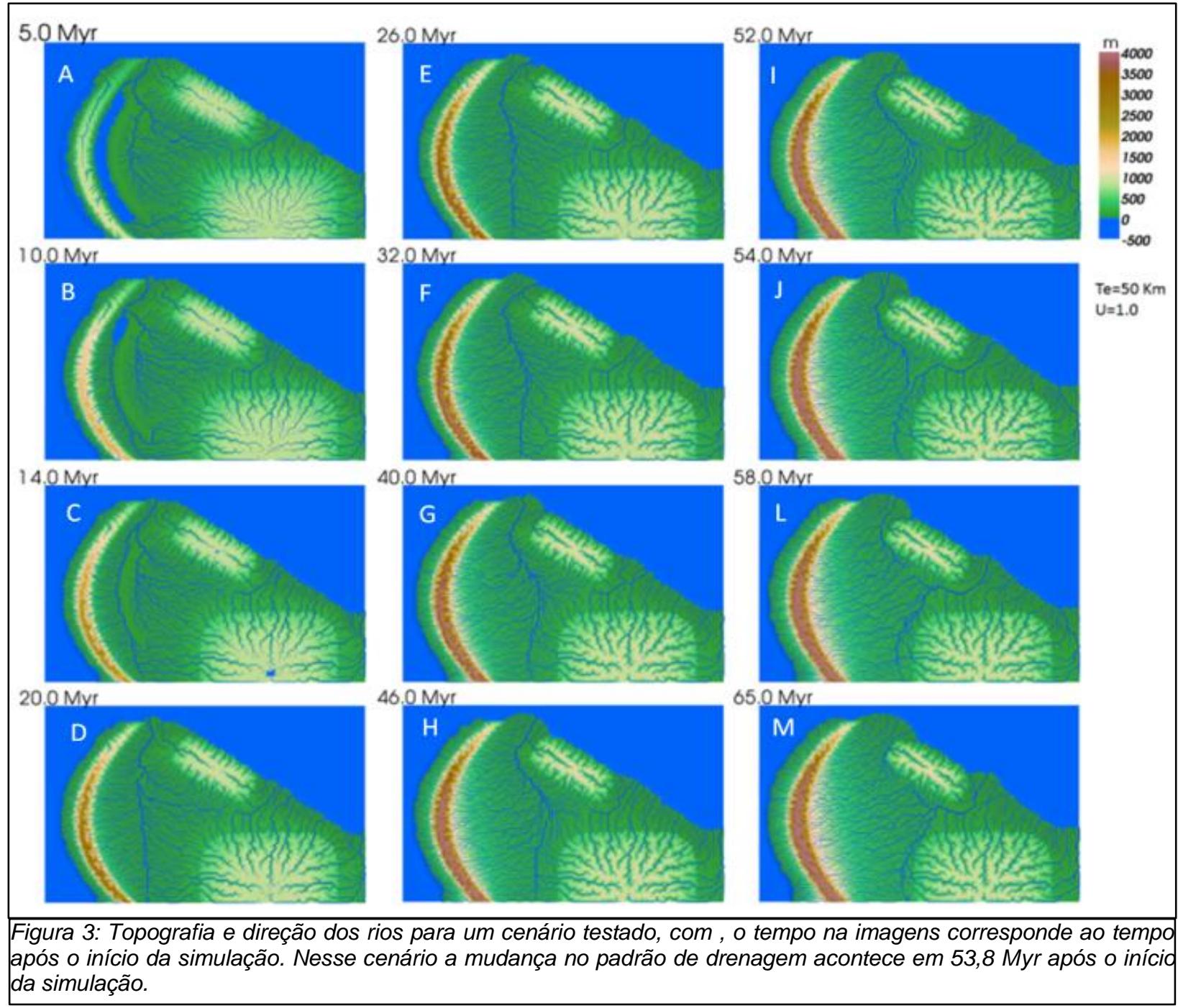

No cenário mostrado na Figura 3 a mudança na drenagem ocorre em 53.8 Myr após o início da simulação com a captura da parte sul do canal axial (veja Figura 3 I, J), essa é a data que ocorre a maior variação na taxa de deposição na foz do Amazonas, como mostra o gráfico na Figura 4A. Após a mudança na drenagem a bacia hidrográfica que agora é drenada para o Atlântico passa a captar cada vez mais afluentes com origem na parte norte dos Andes, o que gera um contínuo aumento na taxa de sedimentação mesmo após a reversão, como mostra a Figura 4A e 4B.

A partir da Figura 4A e 4B e da Tabela 2, nota-se que a data de reversão é maior para $T_{e}$ maiores, isso acontece pois a largura das bacias de ante-país aumenta, e o espaço de acomodação para sedimentos também é maior. Dessa maneira, o tempo que leva para esse espaço ser preenchido aumenta, o que atrasa o início da deposição de sedimentos andinos na Bacia do Solimões e consequentemente o tempo para a topografia nessa região superar o divisor de drenagem.

Para taxas de espessamento crustal maiores o tempo na reversão da drenagem é menor, ou seja, quanto mais rápido se dá o soerguimento da cordilheira mais rápido ocorre a mudança na drenagem.

As datas da mudança na drenagem para os outros cenários testados estão na Tabela 2. Essa foi a data em que houve o maior aumento na taxa de sedimentação na foz do Amazonas.

Tabela 1:Data da mudança na drenagem após o início da simulação para todos os cenários testados.

\begin{tabular}{|c|c|c|c|c|}
\hline \multirow{2}{*}{$\begin{array}{c}\text { U } \\
100 \%\end{array}$} & $50 \mathrm{~km}$ & $60 \mathrm{~km}$ & $70 \mathrm{~km}$ & $80 \mathrm{~km}$ \\
\cline { 3 - 5 } & & & & \\
\cline { 2 - 5 } $300 \%$ & $48.8 \mathrm{Myr}$ & $57.0 \mathrm{Myr}$ & $58.8 \mathrm{Myr}$ & $62.4 \mathrm{Myr}$ \\
\cline { 2 - 5 } & $40.4 \mathrm{Myr}$ & $51.4 \mathrm{Myr}$ & $55.2 \mathrm{Myr}$ & $53.2 \mathrm{Myr}$ \\
\hline
\end{tabular}

Devido as altas taxas de deposição sedimentar na Bacia do Solimões, que permacene maior que na Foz do Amazonas mesmo após a mudança na drenagem, a 
topografia nessa região assume valores muito elevados, que não são coerentes com o real.

A Figura 5 traz um gráfico com a taxa média de deposição sedimentar na foz do amazonas, antes e depois da mudança no padrão de drenagem. Observa-se que a ordem de grandeza da taxa de sedimentação no leque do Amzazonas predita pelo modelo está próximo do valor calculado por Watss et al. (2009).

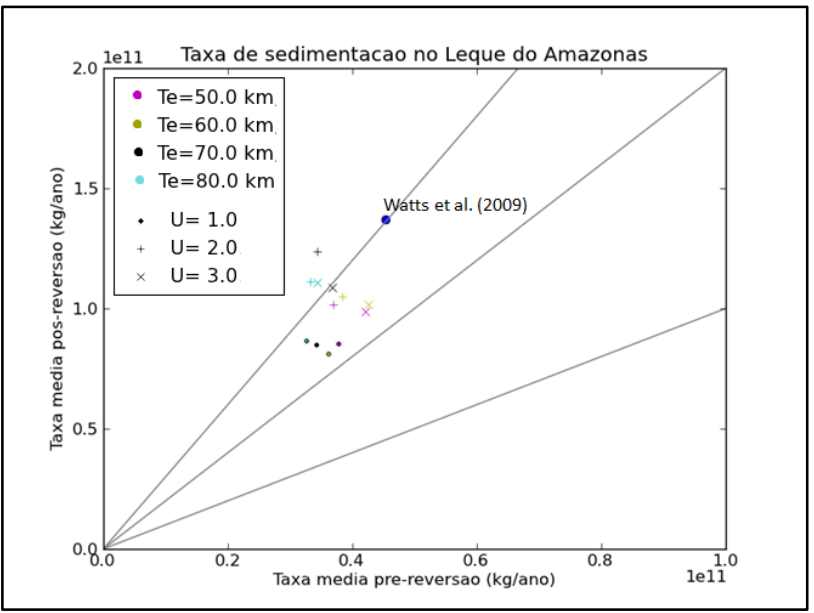

Figura 5:Gráfico com a taxa de sedimentaçãona foz do Amazonas antes e depois da mudança no padrão de drenagem.Os valores mostrados são as médias de taxa de sedimentação durante $10 \mathrm{Ma}$ antes e depois da mudança. O ponto azul representa o valor calculado por Watss et al., (2009). As retas cinzas têm derivada 1, 2 e 3 e servem apenas como referência.

\section{Conclusões}

Apenas com a combinação de orogenia, flexura e isostasia, e processos superficias, foi possível observa a mudança no padrão de drenagem no Norte da América do sul, como Sacek (2014) já havia observado testando outros cenários.

Os testes realizados mostram que a data de mudança na drenagem pode variar com a espessura elástica efetiva da listosfera e também com o aumento da taxa de espessamento crustal na região andina.

Nossas simulações não suportam um aumento significativo na taxa de sedimentação na Foz do Amazonas antes da mudança no padrão de drenagem, como proposto por Latrubesse et al. (2010).

Nossos testes suportam a hipóstese de Figueiredo et al. (2009) que propõe que o aumento da taxa de sedimentação observado no final do Mioceno corresponde a formação do Rio Amazonas como um rio transcontinental.

Nesses testes também não foi observado nenhuma grande zona alagada no oeste Amazônico antes da mudança no padrão de drenagem, o que seria correspondente ao Sistema Pebas (Hoorn et al. 2010). No entanto, como já discutido, a topografia no oeste amazônico ao final das simulações assume valores muito elevados, e isso pode evitar a formação de um grande lago.

Agradecimentos (Font: Arial Bold, 9)

Agradeço 0 apoio financeiro oferecido para o desenvolvimento desse trabalho pela agência de fomento CAPES (Coordenação de Aperfeiçoamento de Pessoal de Nível Superior) através de bolsa de mestrado.

\section{Referências}

Figueiredo J.P., Hoorn C., van der Ven P., Soares E., 2009, Late Miocene onset of the Amazon River and the Amazon deep-sea fan: Evidence from the Foz do Amazonas Basin, Geology, vol. 37, p. 619-622.

Hoorn C., Wesselingh F. P., ter Steege H., Bermudez M. A., Mora A., Sevink J., Sanmartín I., Sanchez-Meseguer A., Anderson C. L., Figueiredo J. P., Jaramillo C., Riff D., Negri F. R., Hooghiemstra H., Lundberg J., Stadler T., Särkinen T., Antonelli A., 2010, Amazonia through time: Andean uplift, climate change, landscape evolution, and biodiversity. Science (New York, N.Y.), vol. 330, p. 927931.

Latrubesse, E.M., Cozzuol, M., Silva-Caminha, S.A.F. Rigsby, C.A., Absy, M.L., Jaramillo, C., 2010, The Late Miocene paleogeography of the Amazon Basin and the evolution of the Amazon River system. Earth and Science Reviews, vol 99, p 99-124.

Sacek, V. \& Ussami, N., 2009, Reappraisal of the effective elastic thickness for the sub-Andes using 3-D finite element flexural modelling, gravity and geological constraints. Geophysical Journal International, vol 179, pg. 778-786.

Sacek, V., 2014, Drainage reversal of the Amazon River due to the coupling of surface and lithospheric processes, Earth and Planetary Science Letters, vol. 401, p. 301-312.

Shephard, G., Müller, R., Liu, L., Gurnis, M., 2010. Miocene drainage reversal of the Amazon River driven by plate-mantle interaction. Nat. Geosci. 3, 870-875.

Wanderley-Filho, J.R., Eiras, J.F., da Cruz Cunha, P.R., van der Ven, P.H., 2010. The Paleozoic Solimões and Amazonas basins and the Acre foreland basin of Brazil. In: Amazonia: Landscape and Species Evolution: A Look into the Past. Wiley Online Library, pp. 29-37.

Watts, A.B., Rodger, M., Peirce, C., Greenroyd, C., Hobbs, R., 2009. Seismic structure, gravity anomalies, and flexure of the Amazon continental margin, NE Brazil. Jounal of Geophysical Research. 114, B07103. 